\title{
Kommunikation mit Patienten und Angehörigen nach einem Behandlungszwischenfall
}

Olga Frank

Korrespondenz:

Olga Frank

Diplom-Pflegewirtin

Stiftung für Patientensicherheit

Asylstrasse 41

CH-8032 Zürich

Tel. 0432437672

Fax 0432437671

frank@patientensicherheit.ch

www.patientensicherheit.ch

\section{Einleitung}

Eines der wichtigsten gesundheitspolitischen Themen in der heutigen Gesundheitsversorgung ist die Patientensicherheit. Fehler in der Gesundheitsversorgung gehören zu den 10 häufigsten Todesursachen [1]. Diese Zahl ist dramatisch und unterstreicht die zwingende Notwendigkeit zu handeln. Dabei ist wichtig zu wissen, dass es wirksame Möglichkeiten gibt, Risiken zu minimieren und aus Fehlern zu lernen. Leider wird dieses Wissen bisher noch viel zu wenig genutzt, geschweige denn, systematisch angewandt.

Missverständnisse, Fehlinterpretationen und Fehlbeurteilungen sind die häufigsten Formen des Scheiterns der Verständigung zwischen den Behandelnden und den Patienten. Der Umgang mit der Situation, insbesondere die richtige Kommunikation nach einem Zwischenfall, ist daher zentraler Aspekt im Themenkreis «Patientensicherheit, klinisches Risikomanagement und Betreuungsqualität».

Die Stiftung für Patientensicherheit der Schweiz hat sich dem Thema angenommen und in enger Zusammenarbeit mit Experten verschiedener Fachgesellschaften und -organisationen einen Flyer entwickelt, der die Gesundheitsinstitutionen und der in der Gesundheitsversorgung tätigen Personen bei der Erstellung ihrer betriebsinternen Richtlinien unterstützen soll.

\section{Patientensicherheit in einer}

\section{konstruktiven Unternehmenskultur}

In unserer Gesellschaft haben die meisten Menschen Angst, einen Fehler zu machen und diesen eingestehen zu müssen. Besonders in der heute hochtechnisierten Medizin können Fehler fatale Behandlungszwischenfälle zur Folge haben. Diese Behandlungszwischenfälle sind dann für alle Beteiligten eine aussergewöhnliche Belastung. Die Ärzte, Pflegenden und Therapeuten stehen daher unter einem enormen Leistungsdruck, keine Fehler machen zu dürfen. Dieses Verhalten basiert besonders in der Medizin auf traditionell verankerte autoritäre Denkweisen und auf eine klar strukturierte und manifestierte Hierarchie in den Gesundheitsinstitutionen.

\section{Communication avec le patient} et ses proches après un incident

\section{de traitement}

Les réactions du patient et de ses proches à un incident sont influencées autant par l'événement luimême que par la manière dont il est pris en compte par l'équipe médicale. Une gestion inadéquate ou un manque de sensibilité conduit à un traumatisme émotionnel supplémentaire. A I'inverse, la reconnaissance honnête du dommage subi, les témoignages d'empathie, une bonne communication et une application efficace de mesures correctives sont de nature à en atténuer les répercussions.

La gestion d'un incident survenant lors d'un traitement est au centre d'une culture de la sécurité qui fixe la manière de réagir et de communiquer en pareil cas. Le patient victime d'un dommage a des attentes claires en termes de communication. II veut:

- $\quad$ recevoir une information rapide sur ce qui s'est concrètement passé;

- ressentir les regrets sincères des soignants;

- connaître les conséquences précises de l'incident sur la suite du processus de traitement;

- entendre que l'établissement a tiré des enseignements de son erreur;

- apprendre où il pourra trouver de l'aide et quelles solutions se présentent à lui.

La confiance dans l'équipe soignante et dans l'établissement ne peut être conservée que dans la mesure où toutes ces informations sont communiquées de façon correcte. La plus grande ouverture face aux incidents est en effet indispensable à la compréhension et à la transparence. 
Der Umgang mit Fehlern hat sich in der Vergangenheit oft darauf beschränkt, nach einem Schuldigen für den aufgetretenen Fehler zu suchen, diesen blosszustellen und zu sanktionieren. Dieses Vorgehen behindert nach wie vor einen konstruktiven Umgang mit Fehlern im Sinne der Aufhebung des klassischen «OpferTäter»-Denkens. Wenn wir wegkommen von der Frage: «Wer war das?» und uns der Frage zuwenden: «Warum und wie konnte das geschehen?», dann ist das der richtige Schritt auf dem Weg von einer «Blame-Kultur» zu einer «Sicherheitskultur». Ein konstruktiver Umgang mit Fehlern und eine nachhaltige Veränderung der Unternehmenskultur würden nicht nur der betroffenen Person helfen, sondern vor allem auch dem Patienten und seinen Angehörigen. In einer Sicherheitskultur müssen Fehler sanktionsfrei aufgenommen, als Lernchance erkannt und für Veränderungen und Weiterentwicklungen in den Unternehmensprozessen genutzt werden.

\section{Stiftung für Patientensicherheit der Schweiz}

Die Stiftung für Patientensicherheit der Schweiz ist im Jahre 2003 auf Initiative des Bundes, der Schweizerischen Akademie der Medizinischen Wissenschaften, den Gesundheitsberufsverbänden, dem Kanton Tessin und der Schweizerischen Patientenorganisation gegründet worden. Sie hat als nationale Plattform zum Ziel, Aktivitäten, Methoden und Projekte zur Verbesserung und Förderung der Patientensicherheit in Schweizer Gesundheitsinstitutionen zu entwickeln, zu unterstützen und zu koordinieren. Dafür arbeitet sie in Netzwerken mit Stiftungsträgern, Leistungserbringern, Verbänden, Behörden, Experten und Patientenorganisationen zusammen.

Die Stiftung für Patientensicherheit erkannte das Thema «Kommunikation mit Patienten und Angehörigen nach einem Zwischenfall» als einen zentralen Brennpunkt in der Gesamtdiskussion um Behandlungszwischenfälle in Gesundheitsinstitutionen. Deshalb griff sie gemeinsam mit der Schweizerischen Gesellschaft für Anästhesiologie und Reanimation (SGAR) dieses Thema auf und entwickelte einen Flyer, der die wichtigsten Aspekte bei der Kommunikation nach einem Behandlungszwischenfall mit anschaulichen Illustrationen und Texten zusammenfasst.

\section{Kommunikation als zentraler Aspekt der Patientensicherheit}

Kommunikation ist die Basis von sozialen Systemen unserer Gesellschaft. Eines ist klar: Wo Menschen arbeiten, passieren auch Fehler. Fehler in
Gesundheitsinstitutionen haben oft dramatische Folgen für alle Betroffenen. Häufig lassen sich diese Fehler auf Schwächen in der Kommunikation zwischen den am Behandlungsprozess beteiligten Personen zurückführen. Daraus wird ersichtlich, welchen Stellenwert die Kommunikation generell und im besonderen im Hinblick auf die Patientensicherheit hat.

Studien zeigen, dass betroffene Patienten ein Interesse daran haben, über Behandlungsfehler informiert und aufgeklärt zu werden. Auch die Öffentlichkeit fordert eine vertrauensvolle Kommunikation zwischen Behandelnden und betroffenen Patienten [2]. Der Patient hat einen Anspruch darauf, über alle Risiken und alle Behandlungsalternativen informiert $\mathrm{zu}$ werden. Wenn sich alle am Behandlungsprozess beteiligten Personen bemühen, offen mit dem Patienten zu kommunizieren, und so die Grundlage dafür schaffen, dass der Patient ihnen auch in kritischen Situationen vertraut, so entspricht das nicht nur den Idealen einer professionellen Behandlung, sondern senkt auch die Wahrscheinlichkeit, dass die Behandelnden auf juristischem Weg zur Rechenschaft gezogen werden [3].

\section{Kommunikation mit Patienten und Angehörigen nach einem Zwischenfall}

Ist der Patient durch einen Behandlungszwischenfall zu Schaden gekommen, hängt für die weitere Behandlung und für das Vertrauensverhältnis viel davon $a b$, ob und wie sich die am Behandlungsprozess beteiligten Personen in dieser schwierigen Situation verhalten. Patienten haben nach einem Behandlungszwischenfall klare kommunikative Anliegen. Wenn diesem Anliegen nicht in der richtigen Art und Weise nachgegangen wird und Informationen zurückgehalten bzw. verschwiegen werden, wird das Vertrauen des Patienten gegenüber dem Behandlungsteam und der Gesundheitsinstitution darunter leiden. Ein offener Umgang mit Behandlungszwischenfällen ist demzufolge von grosser Bedeutung. Eine mitfühlende und ehrliche Patientenaufklärung schafft eine Atmosphäre des Vertrauens und ist somit die Voraussetzung für eine konstruktive Bewältigung von Behandlungszwischenfällen.

Das in den USA erschienene Dokument «When Things Go Wrong» [4] formuliert konkrete Empfehlungen beim Umgang nach einem Behandlungszwischenfall. In Anlehnung an dieses von den Harvard-Spitälern entwickelte Vorgehen hat die Stiftung für Patientensicherheit das Vorgehen adaptiert und einen Flyer entwickelt, der die zentralen Aspekte bei der Kommunikation mit Patienten und Angehörigen 
nach einem Behandlungszwischenfall prägnant zusammenfasst und mit eindrücklichen Illustrationen untermalt. Nachfolgend werden die Empfehlungen der Stiftung für Patientensicherheit vorgestellt.

\section{Bewahrung vor weiterem Schaden}

Erstes Gebot nach einem Behandlungszwischenfall ist die Bewahrung des Patienten vor weiterem Schaden. Dazu bedarf es eines koordinierten und verantwortungsbewussten Vorgehens durch die Unternehmensführung [5].

\section{Wann?}

Passiert ein Zwischenfall, soll nach Vorliegen von verlässlichen Informationen und klaren Fakten das Gespräch mit dem Patienten so rasch als möglich, möglichst innerhalb von 24 Stunden, gesucht werden. Die frühzeitige Kommunikation mit dem Patienten und seinen Angehörigen ist für den Erhalt des Vertrauens wesentlich.

\section{Wer?}

Das Gespräch ist von einer verantwortlichen Person aus dem Behandlungsteam zu führen, zu der der Patient ein Vertrauensverhältnis hat und durch die das Gefühl von Fürsorge, Sorge und Kompetenz in der weiteren Behandlung vermittelt wird.

\section{Wo?}

Die Wahl einer geeigneten Umgebung, in der das Gespräch geführt wird, ist wichtig. Dabei sollte berücksichtigt werden, dass die Privatsphäre des Patienten geschützt wird und eine ruhige Kommunikation möglich ist.

\section{Was soll in einem Gespräch mit dem Patienten und seinen Angehörigen kommuniziert werden?}

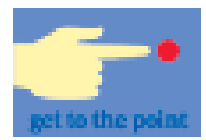

Die ersten Erklärungen sollten sich auf Fakten beschränken, die Hinweise darauf geben, was konkret und wie etwas passiert ist und welche unmittelbaren Auswirkungen dieser Behandlungszwischenfall für den weiteren Behandlungsverlauf hat.

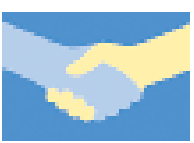

Von entscheidender Bedeutung für das Aufrechterhalten des Vertrauensverhältnisses ist, dass von seiten der Behandelnden ein Bedauern zum Ausdruck gebracht wird.

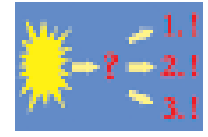

Darüber hinaus sollten die medizinischen Folgen dieses Behandlungszwischenfalls aufgezeigt und gemeinsam mit dem Patienten und seinen Angehörigen mögliche Behandlungsstrategien zur Bewältigung dieser medizinischen Folgen besprochen werden.

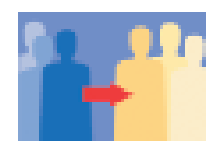

Ist das Vertrauensverhältnis nach einem Behandlungszwischenfall gestört, sollte ein alternatives Behandlungsteam zur Verfügung stehen. Auch im Falle eines ungestörten Vertrauensverhältnisses zwischen den Behandelnden und dem Patienten ist das Angebot eines solchen Teams ratsam. Die Entscheidung tragen dabei allein der Patient und seine Angehörigen.

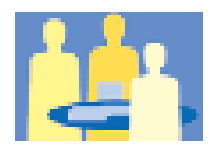

Geschädigte Patienten haben ein grosses Interesse daran, zu sehen, dass ihr Erlebtes eine positive Wirkung auf derartige Ereignisse in Zukunft haben wird. Das Aufzeigen, dass und wie die Gesundheitsinstitution aus dem Behandlungszwischenfall lernen wird, hilft dem Patienten und seinen Angehörigen bei der Bewältigung des Geschehenen.

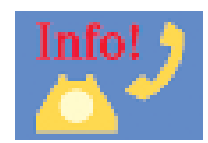

Für die abschliessende Bewältigung des Behandlungszwischenfalls ist die regelmässige Information des Patienten über neue Erkenntnisse unerlässlich. Nur durch die Aufrechterhaltung des Vertrauens, auch nach dem Aufenthalt in einer Gesundheitsinstitution, erfolgt die Nachsorge in einer professionellen und geeigneten Art und Weise.

\section{Weitere wichtige Aspekte nach einem Behandlungszwischenfall}

Für ein erfolgreiches Management eines Behandlungszwischenfalls sind ein institutioneller Rahmen und die Unterstützung durch die Führungsetage und eine entsprechende Unternehmenskultur in der Gesundheitsinstitution erforderlich. Schwerwiegende Behandlungszwischenfälle sind Chefsache. Dieser verantwortet die Koordination der Aktivitäten zur Analyse des Behandlungszwischenfalls und trägt Sorge dafür, dass daraus entwickelte Verbesserungsund Lernprozesse in der Gesundheitsinstitution umgesetzt werden. Voraussetzung für eine lückenlose Analyse des Behandlungszwischenfalls ist 
zum einen die Sicherstellung aller Dokumente, Akten, benutzter Medikamente, gebrauchter Verbrauchsmaterialien (inkl. Abfälle) sowie aller benutzter Geräte, und zum anderen, die Erstellung eines zeitnahen Gedächtnisprotokolls aller Beteiligten am Behandlungszwischenfall. Für die Kommunikation gegenüber der Öffentlichkeit und innerhalb der Gesundheitsinstitution ist eine Strategie festzulegen. Alle Aktivitäten sind für die interne Qualitätsverbesserung zu dokumentieren.

\section{Die Empfehlungen der Stiftung für Patientensicherheit}

Die Stiftung für Patientensicherheit Schweiz bearbeitet in enger Zusammenarbeit mit Experten regelmässig unterschiedliche Themenschwerpunkte und entwickelt daraus Empfehlungen, die bei der Erstellung betriebsinterner Richtlinien für alle an dem Behandlungsprozess beteiligten Personen in verschiedenen Gesundheitsinstitutionen als Unterstützung dienen sollen. Die spezifische Ausgestaltung, Anwendung und betriebsindividuelle Adaption dieser Empfehlungen liegt in der Eigenverantwortung der Gesundheitsinstitutionen.

Die Empfehlungen zur Kommunikation mit Patienten und Angehörigen nach einem Zwischenfall hat die Stiftung für Patientensicherheit in einem Flyer prägnant zusammengefasst. Weiterführende Literatur findet sich im Originaldokument «When Things Go Wrong». Dank der Übersetzungserlaubnis der Autorenschaft ist eine deutschsprachige Version «Wenn etwas schief geht» bei der Stiftung für Patientensicherheit erhältlich [6]. Dieses dient als praxisorientiertes Handbuch zur vertieften Auseinandersetzung mit dem Thema Kommunikation mit
Patienten und Angehörigen nach einem Zwischenfall.

Da gerade das Thema Kommunikation ein interdisziplinäres Thema ist und alle am Behandlungsprozess beteiligten Personen gleichermassen betrifft, möchte die Stiftung den Flyer und auch das Hintergrunddokument so breit als möglich in den Gesundheitsinstitutionen verbreiten. Darüber hinaus wird die Stiftung für Patientensicherheit die Kommunikation nach einem Behandlungszwischenfall an ihrer Tagung am 13. und 14. September 2007 thematisieren und praxisorientierte Workshops anbieten. Die ernsthafte Auseinandersetzung mit diesem Thema kann bereits dazu beitragen, einen offenen, konstruktiven Umgang mit Behandlungszwischenfällen und eine angstfreie Kultur zu schaffen. Nicht nur zum Wohle der Patienten, sondern auch zum Wohle der am Behandlungsprozess beteiligten Personen einer Gesundheitsinstitution.

\section{Literatur}

1 Holzer E, Thomeczek C, Hauke E, Conen D, Hochreutener MA. Patientensicherheit. Wien: Facultas; 2005.

2 Mazor KM, Simon SR, Gurwitz JH. Communicating with patients about medical errors. Arch Intern Med. 2004;164:1690-97.

3 Australian Council for Safety and Quality in Health Care. Open disclosure standard, July 2003. www.safetyansquality.org.

4 When Things go Wrong. Responding to Adverse Events. A Consensus Statement of the Harvard Hospitals. March 2006. www.macoalition.org.

5 American Society for Healthcare Risk Management. Perspective on disclosure of unanticipated outcome information. 2001. www.ashrm.org.

6 www.patientensicherheit.ch/de/projekte/flyer kommunikation_d.pdf. 